\title{
The relationship between EUV dimming and coronal mass ejections
}

\section{Statistical study and probability model ${ }^{\star}$}

\author{
D. Bewsher ${ }^{1}$, R. A. Harrison ${ }^{1}$, and D. S. Brown ${ }^{2}$ \\ 1 Space Science and Technology Department, STFC Rutherford Appleton Laboratory, Chilton, Didcot, Oxfordshire OX11 0QX, UK \\ e-mail: d.bewsher@rl.ac.uk \\ 2 Institute of Mathematical and Physical Sciences, Aberystwyth University, Penglais, Aberystwyth, Ceredigion SY23 3BZ, UK
}

Received 5 September 2007 / Accepted 30 November 2007

\begin{abstract}
Aims. There have been many studies of extreme-ultraviolet (EUV) dimming in association with coronal mass ejection (CME) onsets. However, there has never been a thorough statistical study of this association, covering appropriate temperature ranges. Thus, we make use of a large campaign database utilising the Coronal Diagnostic Spectrometer (CDS) and the Large Angle and Spectrometric COronagraph (LASCO) both on the SOlar and Heliospheric Observatory (SOHO) to associate dimming events detected at 1 and 2 million $\mathrm{K}$ with CME activity. The aim is to confirm whether the dimming-CME association is real or not. This in turn will confirm whether special attention should be paid to the EUV dimming in the pre-eruption and eruption periods to study the CME onset process itself.

Methods. The CDS CME onset campaign data for Mg IX and Fe XVI observations on the solar limb are used to compare to LASCO event lists over a period from 1998 to 2005. Dimming events are identified and the physical extent explored, whilst comparing the events to overlying CME activity.

Results. For the identified dimming regions we have shown strong associations with CME onsets, with up to 55\% of the dimming events being associated with CME activity. This is compared to the random case where up to $47 \%$ of the dimming regions are expected to be associated with CMEs. We have also shown that up to $84 \%$ of CMEs associated with our data can be tracked back to dimming regions. This compares to a random case of up to $58 \%$.

Conclusions. These results confirm the CME-EUV dimming association, using a statistical analysis for the first time. We discuss the repercussions for the study of $\mathrm{CME}$ onsets, i.e. analysis of the dimming regions and the periods up to such dimming may be key to understanding the pre-CME onset plasma processes. The results stress that one emission line may not be sufficient for associating dimming regions with CMEs.
\end{abstract}

Key words. Sun: coronal mass ejections (CMEs)

\section{Introduction}

Coronal mass ejections (CMEs) are dramatic events when seen in white light coronagraph images. Observing the initiation of a CME in the low corona in the X-ray or extreme-ultraviolet (EUV) regimes, below the fields of view of coronagraphs, however, is a notoriously difficult task. An event which is readily detected in the outer corona can be almost impossible to detect lower in the solar atmosphere. Clearly, such low-coronal observations relating to $\mathrm{CME}$ activity are extremely important; they can allow us to identify the source region of the CME itself, and can enable studies of the onset process.

Observations of regions of coronal dimming - a reduction in intensity of the low corona - have been associated with CMEs (for example Harrison 2003) and the so-called EIT waves (Biesecker et al. 2002). This has generated some discussion about the relevance of such dimming to CME onsets and the investigation of dimming characteristics to investigate the CME process. It is recognised that coronal dimming may be caused by many mechanisms, some of which may not be

\footnotetext{
* Appendix A is only available in electronic form at
} http: //www . aanda.org associated with CME activity. This could include movement of material out of the field of view, or the cooling and heating of material, leading to emission declining in the observed bandpass (Howard \& Harrison 2004).

Coronal dimming has been studied, in particular during the operation of the SOlar and Heliospheric Observatory (SOHO), since 1995, but several earlier references to the subject exist (Rust \& Hildner 1976; Rust 1983; Watanabe et al. 1992; Harrison 1997; Sterling \& Hudson 1997; Gopalswamy \& Hanaoka 1998; Zarro et al. 1999; Harrison \& Lyons 2000; Harrison et al. 2003), and the dimming phenomenon has been used as a way of identifying the CME source region. It has been suggested that the dimming is direct evidence for the loss of material from the low corona which becomes the CME. Comparisons of estimated mass loss from the corona in specific dimming events and the mass of material in associated CMEs have been used to support this idea (Harrison et al. 2003).

Detecting such CME-related dimmings in the low corona have two practical uses:

(i) Observing a dimming event may be the earliest detection of a CME event, possibly some hours before the CME is detected in coronagraph fields. The dimming can be used as 
a predictor of CME activity. Note that if this can be done on the limb, it can also be done on the solar disk. We may detect EUV dimming to predict Earth-directed CMEs.

(ii) Identifying the source region of the CME can allow studies of the onset process itself. If the dimming region is identified, one can examine the topology and plasma diagnostic measurements of the region prior to the observed dimming. This is critical for understanding the CME onset process and would be an extremely powerful tool for discriminating between CME onset models.

Despite these important aspects of coronal dimming research, and numerous studies of coronal dimming and CMEs, the association between the two phenomena is not yet fully understood. This is well illustrated by the question which we address in this paper, and is essential for clear interpretation and use of the dimming phenomenon, namely,

- how significant is the relationship between coronal dimming and CMEs?

In order to address this, we consider a probability model for the case where there is no relationship and any coincidences are random. This will give us baseline expected coincidences with standard deviations that we can compare to the results of a statistical analysis. Large deviations of the observed coincidences from the expected coincidences will indicate that there is a relationship between dimming regions and CMEs.

The various studies of dimming have made use of imaging and spectroscopy in the EUV and X-ray region and, in recent years, EUV imagers have been used more and more to look for CME-related dimming. Hudson \& Webb (1997) describe classifications for coronal dimmings which are associated with flares, CMEs or X-ray brightenings. Harrison (2003) describes a number of dimming events that are associated with CMEs and compares the mass loss in the dimming region using spectroscopic techniques and comparing them to estimated mass in the associated CME as seen with SOHO/LASCO. More recently, Harrison $\&$ Bewsher (2007) have described in detail a coronal dimming event which had a number of other interesting features associated with it, including a pre-flare ascending loops, a flare, a transient cool loop and a CME.

Despite the wealth of study, however, the dimming phenomenon is not fully characterised. Can we be sure that the dimming is due to mass loss? What temperature plasmas are involved in the dimming and what are the plasma properties of the dimming regions in the periods leading up to the eruption? These questions can only be addressed using spectroscopy, with a wide range of wavelengths (temperatures) available to the observer. In short, we require thorough spectroscopic studies to understand the dimming process fully.

Thus, in this paper, we make use of spectroscopic observations in selected emission lines and perform a statistical study of the dimming phenomenon in association with coincident CME data. Thus, we regard this as a definitive statement on the nature of the dimming-CME relationship and thus on the CME onset process.

In Sect. 2 we describe the observation scheme used, utilising EUV data from the SOHO spacecraft. In Sect. 3, we describe the algorithm developed to detect coronal dimmings and link them to CME events. In Sect. 4.1, a case study is used to show the algorithm at work, in Sect. 4.2 we present the results of our statistical study and in Sect. 4.3 we describe the probability study which is used to provide comparison coincidences to help interpret the results of the statistical study. Finally in Sect. 5 we discuss our results and make our conclusions.

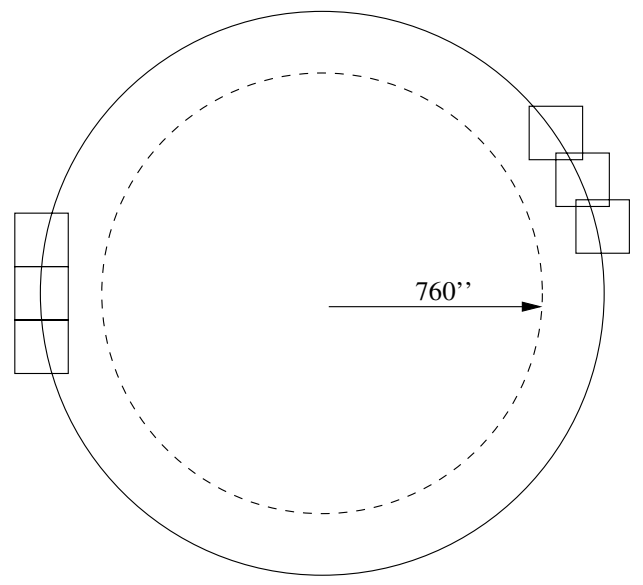

Fig. 1. A schematic diagram illustrating the observing scheme. In each case, the CME onset study was conducted utilising a mosaic of three $4^{\prime} \times 4^{\prime}$ rasters tracing the solar limb region of interest

\section{Observation scheme}

The particular observation scheme utilised to detect and analyse coronal dimming signatures is described in detail by Harrison \& Lyons (2000) and Harrison (2003). These make use of the Coronal Diagnostic Spectrometer (CDS; Harrison et al. 1995) on board the SOHO spacecraft. The basic scheme is the following, and is illustrated schematically in Fig. 1.

The CDS images were made up using a $4^{\prime \prime} \times 240^{\prime \prime}$ slit, with an exposure of $10 \mathrm{~s}$. Interlacing successive exposures with mirror movements was used to build up $4^{\prime} \times 4^{\prime}$ rastered images (with 60 exposures). Three pointings were used in successive rasters to produce a mosaic tracing the limb, as illustrated in Fig. 1. In most cases the sequence was used in an effective $4^{\prime} \times 12^{\prime}$ field of view centred on the equator on the west or east limb (see left hand side of Fig. 1). On occasions, the pointing was designed to trace a higher latitude limb (as shown on the right hand side of Fig. 1). The effective $4^{\prime} \times 12^{\prime}$ field was taken, in each case at a cadence of approximately $50 \mathrm{~min}$. This cadence was dictated by the need to expose for a period able to provide sufficient statistical accuracy and for the need to return a number of spectral emission lines for the required analysis.

The emission line selection was kept to a minimum to minimise the cadence but a wide temperature coverage and some density diagnostic capability was required. Thus, the following lines were returned, their temperatures of maximum abundance given in brackets: He I $584 \AA(20000 \mathrm{~K}), \mathrm{O}$ V $629 \AA(250000 \mathrm{~K})$, Mg IX $368 \AA$ (1 million K), Fe XVI $360 \AA$ ( 2 million K $)$ and Si X $347 / 356 \AA$ ( 1.3 million K). The Si X line pair provides the density diagnostic capability.

In each run of this scheme, observations were usually made over a minimum of several hours. For more details of the observation scheme and the line selection, see Harrison \& Lyons (2000) and Harrison (2003). The scheme, known as the CDS CME onset campaign, has been run on many occasions, as the major component of the so-called SOHO JOP (Joint Observing Programme) 67, since 1996 - the first year of SOHO scientific operation.

A good example of an observing run is shown in Fig. 2. This shows a clear dimming (black region in difference images) on the eastern solar limb under a narrow CME on September 23, 2001 (see Harrison 2006).

The success of this observing scheme in capturing signatures of the CME onset is illustrated by the publication of several 

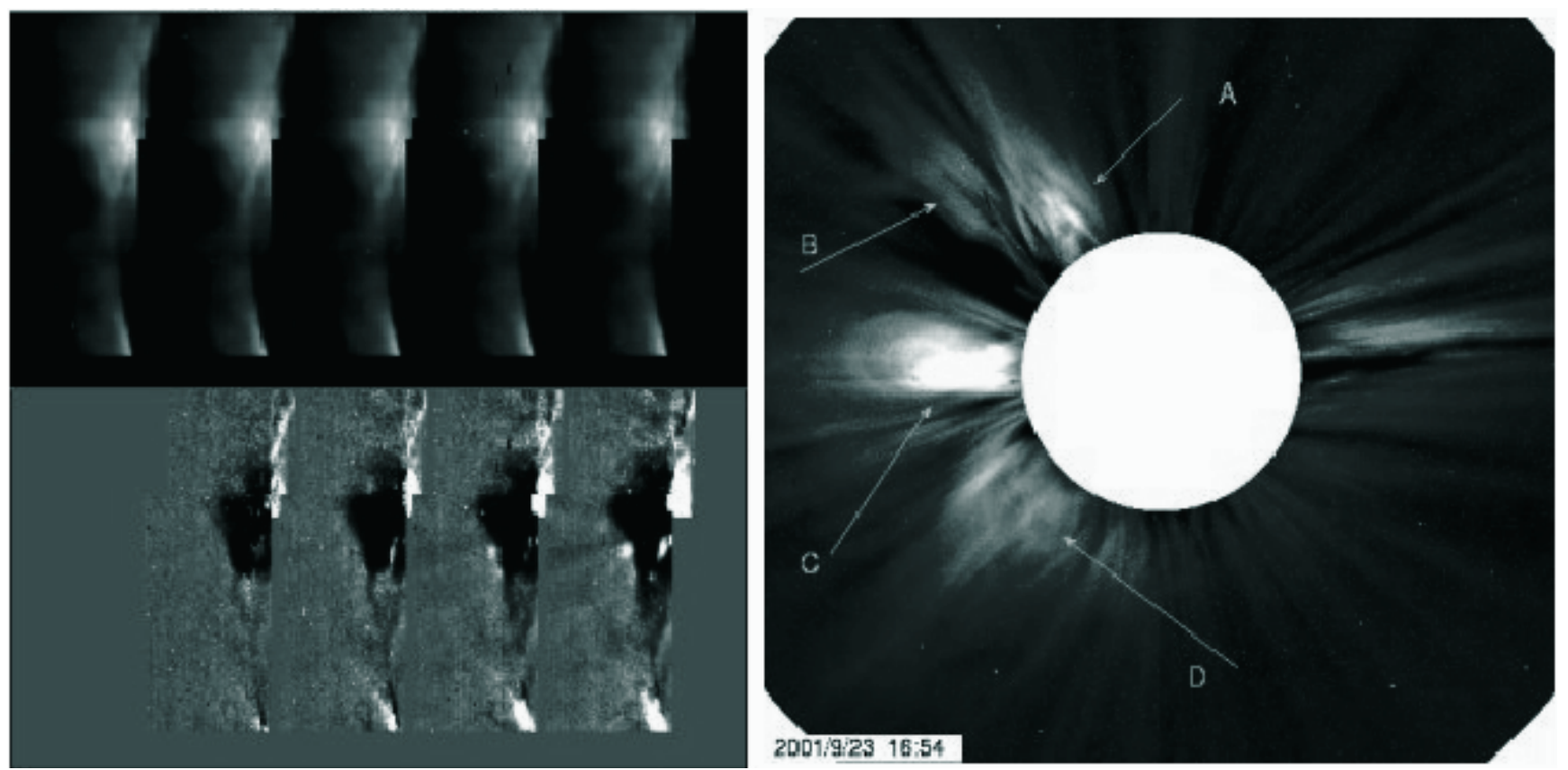

Fig. 2. The event of September 23, 2001. Top left: five CDS mosaics taken in the million K Mg IX $368 \AA$ A emission line on the eastern solar limb. Bottom left: the same images with the first mosaic frame taken from each (fixed difference) showing significant dimming (black) over a portion of the limb. Right: A LASCO coronagraph image of the corona, with the Sun centred under the $4 R_{\odot}$ diameter white disk. Several transient features are identified but the ejection labelled " $C$ " clearly overlies the dimming region and has an identical onset time (see Harrison 2006).

Table 1. Number of SOHO/CDS CME onset studies useful for EUV dimming analysis per year.

\begin{tabular}{ccc}
\hline \hline Year & No. of CDS datasets & $\begin{array}{c}\text { No. of CDS datasets } \\
\text { with LASCO data }\end{array}$ \\
\hline 1998 & 11 & 11 \\
1999 & 22 & 21 \\
2000 & 23 & 22 \\
2001 & 32 & 31 \\
2002 & 26 & 25 \\
2003 & 26 & 26 \\
2004 & 20 & 18 \\
2005 & 14 & 14 \\
\hline Total & 174 & 168 \\
\hline
\end{tabular}

individual event case studies. This includes analyses by Harrison \& Lyons (2000), Harrison (2003), Harrison (2006), Howard \& Harrison (2004), Bewsher \& Harrison (2006) and Harrison \& Bewsher (2007).

\section{A dimming algorithm to provide CME alerts}

\subsection{Selecting CDS studies for analysis}

For this study, we selected all CDS CME onset campaign observations, which have at least five successive mosaic images (i.e. at least 5 mosaics of 3 rastered images each) for which the centre of all of the rasters lies at least 760" from Sun centre (see Fig. 1). We study regions near the limb to ensure that we are best suited to the coronagraph CME images which, due to the geometry of the Thomson scattering process are best suited to observing ejecta in the plane of the sky.

Table 1 shows the number of CDS datasets that meet these requirements. We also identify the number of these datasets for which we have coincident coronagraph data using the LASCO instrument on SOHO (Brueckner et al. 1995). We discuss the specific coronagraph event lists later. The table shows that we have 168 datasets for which we can perform the following analysis. A complete list of the CDS datasets is given in Appendix A.

\subsection{Data manipulation and the identification of dimming events}

Standard software packages have been developed and regularly utilised for CDS data. For the CDS CME onset datasets, we correct the data for missing pixels, for CCD readout bias, for cosmic ray hits, flat fielding and, finally, apply a calibration to convert from data units to photon events per pixel per second. The emission line profiles are examined to identify the contribution to the intensity due to the emission line being examined, i.e. the background intensity on which the line sits in each case is subtracted. The statistical error of each pixel is calculated using the equations given in Thompson (1998).

After the preparation, the individual rasters that make up each mosaic are "stuck" together; overlapping rasters and any pointing movements between rasters are taken into account.

At this point, for each observation run, we have a set of mosaics, effectively $4^{\prime} \times 12^{\prime}$ in size for each of the emission lines used in the study. For this study, only emission above the solar limb is considered, so the emission from the solar disk is masked out. Examples of such mosaic sets are shown in Fig. 2 (top left), Figs. $3 \mathrm{a}$ and $4 \mathrm{a}$ as well as in the references given above. We now examine the pixel intensities through the run to look for regions of significant dimming as the run progresses. We do this by taking a fixed difference from each image; the first mosaic is subtracted from the subsequent mosaics in the sequence. Thus, we are using the initial mosaic as a reference frame and regions of enhanced or depleted emission with respect to the 
D. Bewsher et al.: The relationship between EUV dimming and coronal mass ejections. I.
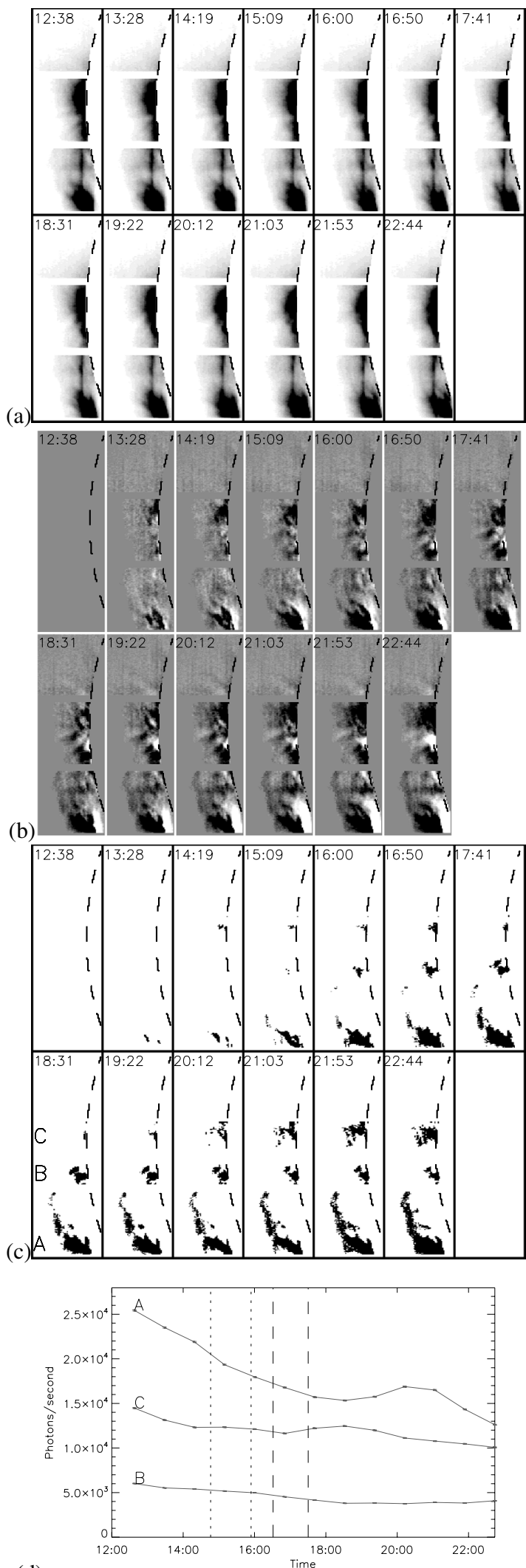

(d)

Fig. 3. a) $\mathrm{Mg} I \mathrm{X}$ data mosaics. b) $\mathrm{Mg} I \mathrm{IX}$ fixed difference mosaics. c) Mg IX dimming regions. The dashed lines mark the position of the solar limb. d) Lightcurve of the dimming region identified in c). Dotted and dashed lines refer to the time when associated CMEs (identified with CACTus and CDAW) were first observed in LASCO and their projected onset time.
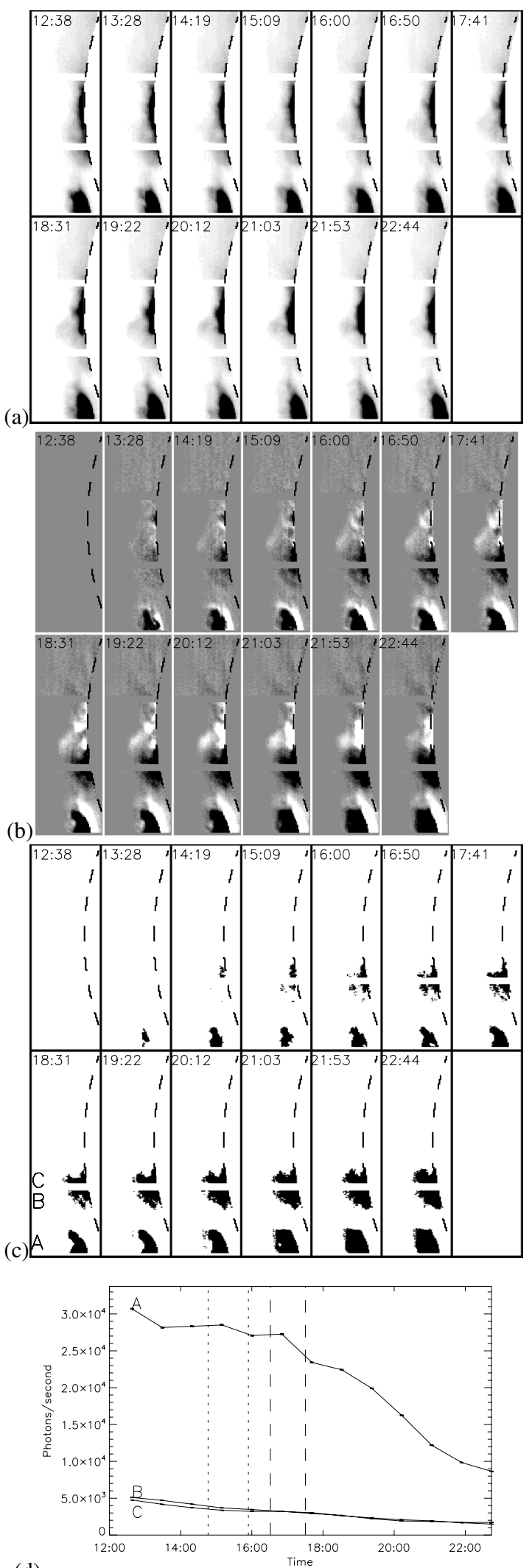

(d)

Fig. 4. a) Fe XIV data mosaics. b) Fe XIV fixed difference mosaics. c) Fe XIV dimming regions. The dashed lines mark the position of the solar limb. d) Lightcurves of the dimming regions identified in c). Dotted and dashed lines refer to the time when associated CMEs (identified with CACTus and CDAW) were first observed in LASCO and their projected onset time. 
initial frame are readily identified as regions of brightening or dimming. Again, examples of such differences sequences are shown in Fig. 2 (bottom left), Figs. 3b and 4b.

In practical terms, we start by examining the behaviour of individual pixels for one of the emission lines. If the fixed difference of a pixel, between the mosaic under examination and the reference mosaic, is greater than twice the error, then we regard it as significant. We note the location of the pixel, in space and time, $(x, y, t)$ and proceed to examine the adjacent pixels in space and time.

Initially, we examine the surrounding pixel group; the pixels $(x, y-1, t),(x-1, y, t),(x+1, y, t),(x, y+1, t)$. If any of these pixels display dimming as well, we examine the adjacent pixels to those pixels and the process is repeated until we establish the extent of the dimming. Similarly, the pixel $(x, y, t)$ is also grouped with pixels $(x, y, t-1)$ or $(x, y, t+1)$ (i.e. the same pixel in the mosaic before or after the original) and a similar process is used to investigate the extent of the dimming in time. A minimum group size of one hundredth of the mosaic area is used to limit the spatial extent. This ensures that a contiguous group of pixels which display a decrease in intensity is identified.

Once the dimming group has been identified, we perform an additional check to ensure that the intensity of the whole group still decreases with time.

In this work, the analysis discussed above has been performed on the CDS CME onset campaign mosaics for both the Mg IX and the Fe XVI emission lines. Of course, any dimming identified in one emission line, which is associated with a particular temperature plasma, may look different from the other emission line, so we may find differing dimming extents or durations.

Any dimming regions in either $\mathrm{Mg}$ or $\mathrm{Fe}$ which overlap in angular space and time are linked together for the purposes of our statistical study.

For associations with the LASCO coronagraph data we need to define the extent and location of the dimming and the timing and duration. In effect, we are defining temporal and spatial windows in which to make associations with CME activity. These windows need to be defined well enough to ensure that we identify associations, recognising that we know nothing about the time-altitude profiles of the associated CMEs under the coronagraph occulting discs, but ensuring that we minimise false associations due to coincidence. Utilising past experience we feel that the following spatial and temporal windows are the most appropriate:

- temporal window: Spanning the duration of the dimming period (or dataset) plus $1.5 \mathrm{~h}$ on either side;

- spatial window: Spans the position angle range of the dimming extent (or dataset field-of-view), plus ten heliographic degrees on either side. The position angle is the angle relative to Sun-centre with north defined as zero degrees, east as 90 degrees, south as 180 degrees, etc.

\subsection{Comparison with CME lists}

We make use of two CME event lists which are created from the LASCO observations. Creating a definitive list of events such as CMEs, or even flares, is somewhat subjective so we have chosen to make use of two comprehensive event lists which use different approaches to event recognition. It is expected that the majority of events will occur in both lists, but some events, such as those which are borderline in intensity or structure, could be picked up by only one technique.
These two event lists are:

- Coordinated Data Analysis Workshop (CDAW) CME list which is maintained on the Internet at http: //cdaw.gsfc.nasa.gov/CME_list/

- Computer Aided CME Tracking (CACTus) CME list at http://www.sidc.be/cactus/

Our study needs to identify positive associations between CMEs and dimming events as well as null associations. Thus, our first step is to identify CMEs whose onsets fall within the spatial and temporal windows defined above and then check for any association with dimming events. We note that any event in the CACTus list which is labelled as "marginal" is not considered.

In each of the CDAW and CACTus lists we find the time of the first appearance of any CME events in the LASCO C2 (at $2 R_{\odot}$ ) field of view whose onset may appear within one of the CDS CME onset campaign periods. The linear speed (CDAW) or median velocity (CACTus) of each CME is given in the event lists and this is used to project the start time of the candidate CME back to the surface of the Sun. If this projected time falls within one of the campaign periods, it is used in this study.

We then compare the CME onset time and location with candidate dimming events in the same campaign period. If the projected CME onset time falls within the time window of a dimming event, as described above, then we proceed to check the consistency of locations of that CME and the dimming event. If the position angle range of the CME and the dimming overlap, then we say that the dimming and the CME are related. In other words, we have established that the CME and the dimming event are apparently associated in space and time.

CME onsets that fall within one of the campaign periods but do not appear within a dimming spatial/temporal window are also noted.

\section{Results}

\subsection{Case study}

To demonstrate the algorithm described in Sect. 3, we study in detail the CDS dataset with study number 21822 which was taken on 4 February 2001.

Figure 3 a shows a sequence of mosaics, as described above, of emission from $\mathrm{Mg}$ IX data, i.e. plasma at 1 million $\mathrm{K}$, for a region on the limb on 4 February 2001. Figure $3 b$ shows the same data-set with a fixed difference; the first mosaic is subtracted from the other mosaics. Black and white regions identify regions of dimming and brightening, respectively. Dimming regions are identified using the scheme described above and are shaded in grey in Fig. 3c. In all of these sequences, time runs from the top left mosaic to the bottom right mosaic, with approximately 50 min cadence. In all frames, the dashed black lines mark the position of the limb. The mosaics are centred on position angle $92^{\circ}$ and they have an angular width of $44^{\circ}$.

In the case of this dataset, the individual rasters did not quite sit immediately adjacent to one another. Horizontal data gaps are seen in the data and fixed difference mosaics, where the original rasters did not completely cover the region of interest. In cases like this, we do not fill the data gaps, by projection for example, so that we do not produce any artificial dimming events. It is worth noting that some dimming events may extend beyond the edges of the mosaic fields of view or into such gaps. This should not influence the results significantly.

Figure $3 \mathrm{~d}$ shows the intensity curves of the three dimming regions identified in Fig. 3c. A decrease in intensity (dimming) 


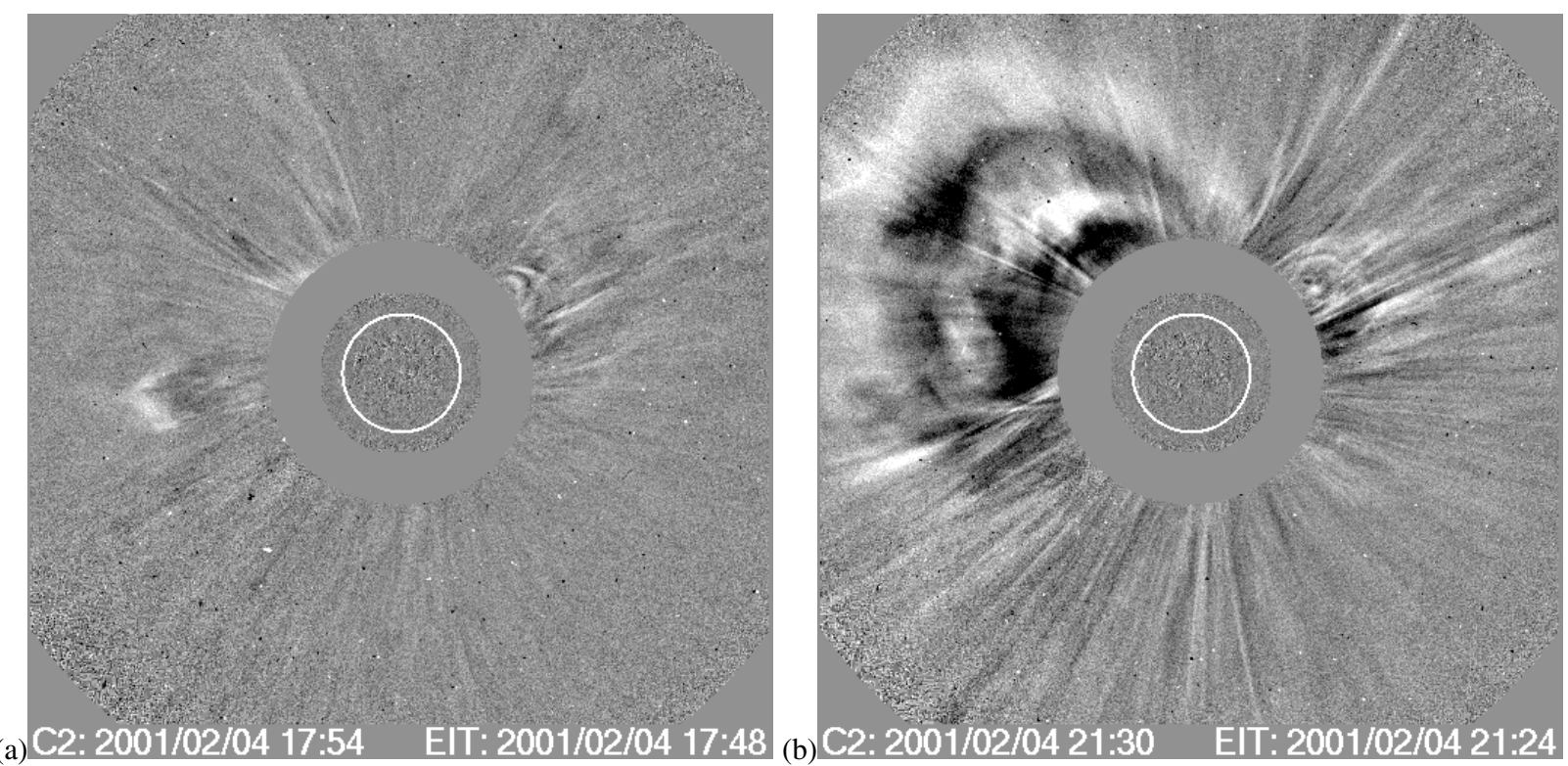

Fig. 5. LASCO C2 difference images showing the two CMEs associated with our dimming. Images courtesy of CDAW.

Table 2. Properties of the dimming regions shown in Figs. 3 and 4d.

\begin{tabular}{cccc}
\hline \hline Dimming & $\begin{array}{c}\text { 1st observed } \\
(\mathrm{UT})\end{array}$ & $\begin{array}{c}\text { Principal } \\
\text { angle }\left({ }^{\circ}\right)\end{array}$ & $\begin{array}{c}\text { Angular } \\
\text { width }\left({ }^{\circ}\right)\end{array}$ \\
\hline Mg IX A & $12: 38$ & 106 & 13 \\
Mg IX B & $12: 38$ & 97 & 5 \\
Mg IX C & $12: 38$ & 88 & 6 \\
Fe XVI A & $12: 38$ & 109 & 5 \\
Fe XVI B & $12: 38$ & 102 & 3 \\
Fe XVI C & $12: 38$ & 97 & 3 \\
\hline
\end{tabular}

can be clearly seen for all three over the time period of the observations.

For the same observations, we also consider the Fe data. Figure 4 shows these observations for the same time and location as for the $\mathrm{Mg}$ observations, and is shown in identical format. Again, clear dimming regions are identified, though one can see that the regions that are identified as dimming do not correspond fully with the $\mathrm{Mg}$ data. The regions labelled A are clearly the same, though the dimming is more extended for the Mg observation. The regions labelled $\mathrm{B}$ and $\mathrm{C}$ in the $\mathrm{Fe}$ data correspond to a more extended version of the region labelled B in the $\mathrm{Mg}$ data. Region $\mathrm{C}$ in the $\mathrm{Mg}$ data did not meet the dimming criteria for the Fe data. This stresses a point made by Harrison et al. (2003), that dimming may occur at different coronal temperatures. This does highlight the value of studying multiple temperatures simultaneously and is one reason why we make use of the $\mathrm{Mg}$ and Fe data in this study.

We are concentrating on the dimming activity in these images but point out that the bright arc in the Fe observation is not significant; its intensity increase is not greater than the statistical error on the count rate.

Table 2 lists the basic parameters of the dimmings identified in these data. To all intents and purposes these dimming events are underway at the start of the observation period (Figs. 3d and $4 d$ ) so the start time is given as the first mosaic. The position angles are calculated and the central position angle of each dimming region (listed as the principal angle) and the angular width are tabulated.
Table 3. Properties of CMEs associated with the coronal dimmings shown in Figs. 3 and 4 d.

\begin{tabular}{ccccc}
\hline \hline CME & $\begin{array}{c}\text { 1st observed } \\
(\mathrm{UT})\end{array}$ & $\begin{array}{c}\text { Onset } \\
(\mathrm{UT})\end{array}$ & $\begin{array}{c}\text { Principal } \\
\text { angle }\left({ }^{\circ}\right)\end{array}$ & $\begin{array}{c}\text { Angular } \\
\text { width }\left(^{\circ}\right)\end{array}$ \\
\hline CACTus A & $15: 54$ & $14: 46$ & 94 & 12 \\
CACTus B & $18: 06$ & $17: 19$ & 44 & 128 \\
CDAW A & $17: 30$ & $16: 31$ & 94 & 24 \\
CDAW B & $17: 54$ & $17: 19$ & 57 & 147 \\
\hline
\end{tabular}

From the table, it is can be seen that the Mg dimming labelled " $A$ " overlaps spatially with the Fe dimmings labelled " $A$ " and "B". For the purposes of our statistical study, these 3 dimming regions are linked together. Also, the $\mathrm{Mg}$ dimming labelled "B" overlaps spatially with the Fe dimming labelled " $C$ ". Similarly, these dimming regions are linked for the statistical study. As mentioned above, there is no corresponding Fe dimming to the $\mathrm{Mg}$ dimming labelled " $\mathrm{C}$ ". All of the dimmings identified in either $\mathrm{Mg}$ or $\mathrm{Fe}$ are temporally associated.

We now examine the CACTus and CDAW CME lists in the time period of this dimming event. Indeed, both the CACTus and CDAW CME lists record two CMEs with start times and locations that meet the criteria given in Sect. 3.2. SOHO/LASCO C2 difference images from the CDAW CME list of the two CMEs identified are shown in Fig. 5. Table 3 gives the start time (time of first appearance in the LASCO/C2 field of view), principal angle and angular width of the CMEs as given in the CDAW and CACTus CME lists. We label the two CME events A and B.

Plane of sky velocities are measured for each event and used to project the onset time. For CME A, the measured velocities were $170 \mathrm{~km} \mathrm{~s}^{-1}$ and $196 \mathrm{~km} \mathrm{~s}^{-1}$ for the CACTus and CDAW lists, respectively. For CME B, the velocities were $248 \mathrm{~km} \mathrm{~s}^{-1}$ and $327 \mathrm{~km} \mathrm{~s}^{-1}$. Clearly there is uncertainty in projecting below occulting discs because we know nothing about the early acceleration of each CME, but the different measured speeds for the same event also stress that there is uncertainty. However, it is clear that the CACTus and CDAW events labelled A are the same and those labelled B are the same. The times of first appearance of the CME in the CACTus and CDAW lists, however, varies by $1 \mathrm{~h}$ and $36 \mathrm{~min}$ for CME "A" and $12 \mathrm{~min}$ for CME "B". The 
Table 4. Overall statistics of dimmings identified in the CDS CME onset observations.

\begin{tabular}{ccccc}
\hline \hline Emission line & $\mathrm{Mg}$ & $\mathrm{Fe}$ & $\mathrm{Mg}$ or Fe & $\mathrm{Mg} \& \mathrm{Fe}$ \\
\hline \# of CDAW CMEs coinciding with datasets & 125 & 125 & 125 & 125 \\
\# of CDAW CMEs coinciding with dimmings & 85 & 82 & 105 & 62 \\
\# of CACTus CMEs coinciding with datasets & 179 & 179 & 179 & 179 \\
\# of CACTus CMES coinciding with dimmings & 102 & 103 & 131 & 74 \\
\hline Total \# of dimmings identified & 155 & 146 & 205 & 96 \\
\# of dimmings associated with CDAW CMEs & 71 & 60 & 85 & 46 \\
\# of dimmings associated with CACTus CMEs & 79 & 72 & 98 & 53 \\
\hline
\end{tabular}

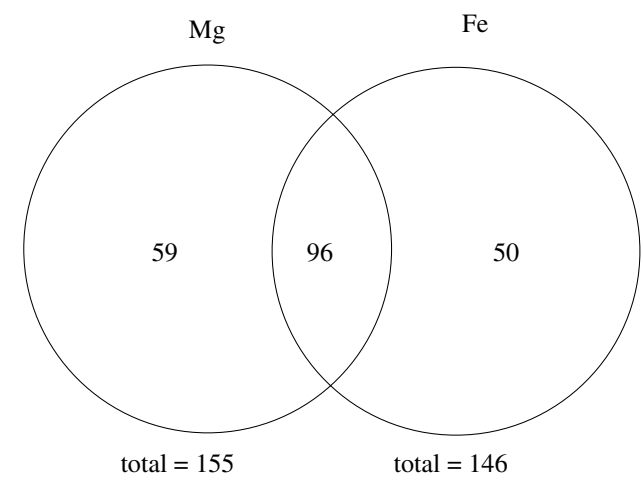

Fig. 6. Venn diagram illustrating which wavelengths the dimming regions were observed in.

estimated onset time of the CMEs using the data from CACTus and CDAW also varies by $1 \mathrm{~h}$ and $45 \mathrm{~min}$ for CME "A".

The CME onset time of 14:46 UT suggested by the CACTus CME "A" data compares the best with the start of the dimmings identified. The CME location is given as $94^{\circ}$ in both cases with spreads of $12^{\circ}$ and $24^{\circ}$; the dimming locations range from $88^{\circ}$ to $109^{\circ}$. The dimming onset at $12: 38 \mathrm{UT}$ is best matched to the 14:46 UT projected onset; this is roughly a $2 \mathrm{~h}$ difference between onset times, though this could be due to the linear CME projection, and the dimming event period encompasses the CME "A" projected onsets from both datasets. Thus, it is very likely that the CME shown in Fig. 5a is the CME associated with our dimmings.

The dotted (dashed) lines in Figs. $3 \mathrm{~d}$ and $4 \mathrm{~d}$ mark the times when the CME seen in Fig. 5a was first observed in the LASCO C2 FOV by the CACTus (CDAW) algorithm, and the estimated onset time of the CME at the solar surface.

\subsection{Statistical study}

Table 4 gives an overview of the dimming events identified in the 168 CDS datasets from 1998 to 2005 . In the statistical study, we have split the dimming regions identified into those that are just identified using only $\mathrm{Mg}$, those that are identified using only $\mathrm{Fe}$, those that are identified in $\mathrm{Mg}$ or $\mathrm{Fe}$ and those that are identified in both $\mathrm{Mg} \& \mathrm{Fe}$. The Venn diagram in Fig. 6 illustrates these different possibilities.

From Table 4 we can see that more dimming regions were identified in $\mathrm{Mg}$ than $\mathrm{Fe}$ and that a large proportion of these dimming regions were identified in both $\mathrm{Mg} \& \mathrm{Fe}$. This implies that identifying dimming regions at a variety of temperatures is essential to accurately determining the nature of the relationship between dimmings and CMEs. It also suggests that one should be careful determining the mass in a dimming region which has only been identified at one temperature. There may be a significant part of the CME that comes from material at a different temperature.

More CMEs identified in the CACTus catalog coincided with both the data sets and the dimming regions than CMEs identified in the CDAW catalogue. Robbrecht \& Berghmans (2004) report that CACTus identifies more CMEs than CDAW. But that some of these CMEs are "gusty outflows" which meet their criteria for a CME. They also mention that CACTus can also split a CME into multiple separate events, where CDAW only registers one event. Hence it is not surprising that we have more CMEs from the CACTus catalog.

The low altitude nature of the dimming inevitably means that we miss dimming events that are occulted by the solar disc, for which there are CMEs identified in the LASCO data. This means that the figures for the number of CMEs coinciding with dimmings given in Table 4 are lower limits. Cases where a dimming region is associated with multiple CMEs are counted only once per dimming region.

\subsection{Probability study}

In order to interpret the results of the statistical study, we consider a probability model of the case that there is no direct link between CMEs and dimming regions and any coincidence between the two is random. This will allow us to judge the significance of the statistical study.

For the probability model, we make the following assumptions:

1. there is no link between CMEs and dimming regions and CMEs may originate from regions of the Sun irrespective of the occurrence of dimming regions;

2. CMEs are independent of other CMEs. The occurrence of CMEs is random in time and space and there is no enforced spreading out, or clustering, of CMEs.

3. all dimming regions are assumed to have the same size (see the definition of dimming region size below), given by the average size of the observed dimming regions in the statistical study.

The first assumption is our null hypothesis. The aim of this probability study is to calculate the number of coincidences you would expect in the random case and compare this to the number of observed coincidences. If the number of observed coincidences is significantly higher than the random case, then we can reject the null hypothesis that dimming regions and CMEs are random and conclude that there is some link between the two.

The second and third assumptions are simplifications to help construct the probability model. The second assumption means that we can work with independent probability events rather than dependent ones - for which we would require an assumption about the distribution of CMEs in time and space anyway. The third assumption is required for the second part of the probability 
model "Probability that a dimming is associated with a CME". This allows us to come up with a "general" figure for comparison rather than having to produce case-specific figures for specific dimming regions in a given distribution. It also allows us to use the binomial distribution rather than a more-involved multinomial distribution.

In the statistical study, CMEs were tracked back to the lower solar atmosphere by eruption time and an angular extent. This was then compared to the extent of the dimming regions in time and angle. Hence, for this probability model, we define the size of a dimming region to be in angle $\times$ time space with units of ${ }^{\circ} h$.

\subsubsection{Probability that a CME is associated with a dimming}

We define the quantity $A_{\text {tot }}$ to be the angle-time volume of all of the $N_{\text {dim }}$ dimming regions in a dataset. The quantity $A_{\text {data }}$ is then defined as the total angle-time volume covered by the dataset. This gives

$p_{\mathrm{c}}=\frac{A_{\mathrm{tot}}}{A_{\text {data }}}$

which is the proportion of the angle-time volume of the dataset occupied by dimming regions.

The average size of a dimming region is given by

$A_{\mathrm{avg}}=\frac{1}{N_{\mathrm{dim}}} \sum_{i} A_{i}=\frac{A_{\mathrm{tot}}}{N_{\mathrm{dim}}}$.

Rearranging gives

$A_{\text {tot }}=N_{\text {dim }} A_{\text {avg }}$

which can be substituted into the above probability equation to give

$p_{\mathrm{c}}=\frac{N_{\mathrm{dim}} A_{\mathrm{avg}}}{A_{\mathrm{data}}}$.

So, if an erupted CME can be traced back to the observed dataset, then it has probability $p_{\mathrm{c}}$ of its origin coinciding with a dimming region. This is taken to be a single trial. As we have assumed that our CMEs are independent from each other, the statistics of multiple CMEs is given by a binomial distribution.

Hence, if there are $N_{\mathrm{CME}} \mathrm{CMEs}$ that are traced back to the observed dataset, then the expected number of CMEs that coincide with a dimming region is given by

$N_{\text {coin }}=N_{\mathrm{CME}} p_{\mathrm{c}}=\frac{N_{\mathrm{CME}} N_{\mathrm{dim}} A_{\mathrm{avg}}}{A_{\text {data }}}$

with standard deviation

$\sigma_{\text {coin }}=\sqrt{N_{\mathrm{CME}} p_{\mathrm{c}}\left(1-p_{\mathrm{c}}\right)}$.

Table 5 shows the trial probabilities $\left(p_{\mathrm{c}}\right)$ calculated for dimmings seen in the $\mathrm{Mg}$ and $\mathrm{Fe}$ lines. The values for $A_{\text {tot }}$ quoted in the table include the $10^{\circ}$ and $1.5 \mathrm{~h}$ buffer zones used in the data analysis. Expected numbers of coincidences are given for CMEs identified in the CDAW and CACTus databases. $N_{\text {coin }}$ gives the predicted number of CME-dimming region coincidences for the observed number of CMEs $\left(N_{\mathrm{CME}}\right)$ as well as a percentile. The standard deviation on the predicted number of CME-dimming region coincidences is also given $\left(\sigma_{\text {coin }}\right)$. The actual number of observed coincidences (as discussed in this paper) is given for comparison $\left(N_{\text {obs }}\right)$.

In all cases, the predicted number of coincidences is dramatically lower than the observed number, with a significance of
Table 5. Results from the probability model considering CME-dimming region coincidences.

\begin{tabular}{lcccc}
\hline \hline Dataset & $\mathrm{Mg}$ & $\mathrm{Fe}$ & $\mathrm{Mg}$ or Fe & $\mathrm{Mg} \& \mathrm{Fe}$ \\
\hline$A_{\text {tot }}\left({ }^{\circ} h\right)$ & 59948 & 55701 & 73098 & 42550 \\
$A_{\text {data }}\left({ }^{\circ} h\right)$ & 126016 & 126016 & 126016 & 126016 \\
$p_{\text {c }}$ & 0.476 & 0.442 & 0.580 & 0.338 \\
$N_{\text {dim }}$ & 155 & 146 & 205 & 96 \\
$A_{\text {avg }}$ & 387 & 382 & 357 & 443 \\
\hline$N_{\text {CME }}(\mathrm{CDAW})$ & 125 & 125 & 125 & 125 \\
$N_{\text {coin }}$ & 59.5 & 55.3 & 72.5 & 42.2 \\
$\sigma_{\text {coin }}$ & 5.6 & 5.6 & 5.5 & 5.3 \\
$N_{\text {obs }}$ & 85 & 82 & 105 & 62 \\
$N_{\text {coin }}(\%)$ & 48 & 44 & 58 & 34 \\
$\sigma_{\text {coin }}(\%)$ & 4 & 4 & 4 & 4 \\
$N_{\text {obs }}(\%)$ & 68 & 66 & 84 & 50 \\
\hline$N_{\text {CME }}(\mathrm{CACTus})$ & 179 & 179 & 179 & 179 \\
$N_{\text {coin }}$ & 85.2 & 79.1 & 103.8 & 60.4 \\
$\sigma_{\text {coin }}$ & 6.7 & 6.6 & 6.6 & 6.3 \\
$N_{\text {obs }}$ & 102 & 103 & 131 & 74 \\
$N_{\text {coin }}(\%)$ & 48 & 44 & 58 & 34 \\
$\sigma_{\text {coin }}(\%)$ & 4 & 4 & 4 & 4 \\
$N_{\text {obs }}(\%)$ & 57 & 58 & 73 & 41 \\
\hline
\end{tabular}

over two standard deviations. This already suggests that the null hypothesis (Assumption 1) is incorrect and demonstrates that CMEs can be tracked backed to dimming regions. For example, $84 \%$ of CMEs associated with our data from the CDAW catalogue track back to a dimming region in either $\mathrm{Mg}$ or Fe lines.

It should be noted that the observed CMEs may originate from outside of the CDS field of view (for example, on the backside of the Sun). If we assume that the CDS dataset gives a representative figure for the proportion of dimming regions to non-dimmed Sun at the observed latitudes, then the probability figures quoted in Table 5 are not unreasonable. However, we might expect the observed number of coincidences $\left(N_{\text {obs }}\right)$ to be higher if we had a more complete observation area.

\subsubsection{Probability that a dimming is associated with a CME}

We now want to turn around the above result and calculate the probability that a dimming region is associated with at least one CME.

From our assumption that all dimming regions are the same size, it is clear that if we have a CME that coincides with a dimming region, then the probability that it is affiliated with a particular dimming region is given by

$P_{\text {affil }}=\frac{1}{N_{\text {dim }}}$

and is the same for any of our $N_{\text {dim }}$ dimming regions.

Given the independence assumption, the number of CMEs affiliated to a particular dimming region is binomially distributed. Hence the probability that a dimming region has 0 CMEs affiliated to it, given $N_{\text {coin }}$ CMEs related to dimming regions and a probability $P_{\text {affil }}$ of a CME being affiliated to the particular dimming region, is given by

$$
\begin{aligned}
P_{0} & =B\left(0, P_{\text {affil }}, N_{\text {coin }}\right) \\
& =\frac{N_{\text {coin }} !}{0 ! N_{\text {coin }} !} P_{\text {affil }}^{0}\left(1-P_{\text {affil }}\right)^{N_{\text {coin }}} \\
& =\left(1-P_{\text {affil }}\right)^{N_{\text {coin }}} .
\end{aligned}
$$


Thus, the probability that a dimming region is associated with at least one CME is given by

$P_{\text {assoc }}=1-P_{0}$.

Hence, for our $N_{\text {dim }}$ dimming regions, the number of dimming regions associated with at least one $\mathrm{CME}$ is given

$N_{\text {assoc }}=N_{\text {dim }} P_{\text {assoc }}=N_{\text {dim }}\left(1-P_{0}\right)$

with a standard deviation of

$$
\begin{aligned}
\sigma_{\text {assoc }} & =\sqrt{N_{\text {dim }} P_{\text {assoc }}\left(1-P_{\text {assoc }}\right)} \\
& =\sqrt{N_{\operatorname{dim}} P_{0}\left(1-P_{0}\right)} .
\end{aligned}
$$

There is a second source of error for this result due to the fact that $N_{\text {coin }}$ is given by a random variable with standard deviation $\sigma_{\text {coin }}$. This is then used in the calculation of $P_{0}$. It is straightforward to show that this error propagates through the calculation of $P_{0}$ to give

$\sigma_{P_{0}}=\left|\ln \left(1-P_{\text {affil }}\right)\left(1-P_{\text {affil }}\right)^{N_{\text {coin }}}\right| \sigma_{\text {coin }}$

which in turn gives an error on $N_{\text {assoc }}$ of

$e_{\mathrm{assoc}}=N_{\mathrm{dim}} \sigma_{p_{0}}$.

We may then add this error to our standard deviation in quadrature to give a total error of

$\delta_{\mathrm{assoc}}=\sqrt{\sigma_{\mathrm{assoc}}^{2}+e_{\mathrm{assoc}}^{2}}$

This quantity should be treated like a total standard deviation, hence observed numbers of coincidences that are more than $2 \delta_{\text {assoc }}$ from the expected value $\left(N_{\text {assoc }}\right)$ are unlikely.

Strictly speaking, $\sigma_{\text {assoc }}$ depends on $\sigma_{P_{0}}$ as well, making $\sigma_{\text {assoc }}$ and $e_{\text {assoc }}$ dependent quantities and leaving us unable to add them in quadrature. However, the dependency is weak for the range of figures that we encounter in this paper, hence the above formula for $\delta_{\text {assoc }}$ is a good approximation. This was confirmed using a Monte Carlo experiment.

Table 6 shows the predicted numbers of dimming regions that are associated with at least one CME $\left(N_{\text {assoc }}\right)$, given the assumptions made, compared to the observed number of coincidences $\left(N_{\text {as-obs }}\right)$. The standard deviations $\left(\sigma_{\text {assoc }}\right.$ and $\left.e_{\text {assoc }}\right)$ are given, along with the total standard deviation $\left(\delta_{\text {assoc }}\right)$. Results are shown as a percentage as well. This is done for CMEs identified in the CDAW and CACTus databases respectively.

From Table 6 we see that in all cases, the predicted number of dimming regions which have at least one associated CME is much less than the observed number. For the CDAW catalogue, this result is significant to at least $2 \delta_{\text {assoc }}$. For the CACTus catalogue, it is significant to $2 \delta_{\text {assoc }}$ for only the $\mathrm{Mg}$ and Fe case. For the other cases, it is significant to at least $1.3 \delta_{\text {assoc }}$.

This answers the question which we posed in the Introduction. There is a significant relationship between dimming regions and CMEs, and the coincidence between the two is not random.

Furthermore, in order to produce a useful algorithm to monitor coronal dimming in the EUV in order to predict CME activity, the algorithm must do better than the 30-47\% figures that the random cases produce.
Table 6. Results from the probability model considering dimming regions that are associated with at least one CME.

\begin{tabular}{lcccc}
\hline \hline Dataset & $\mathrm{Mg}$ & $\mathrm{Fe}$ & $\mathrm{Mg}$ or Fe & $\mathrm{Mg} \mathrm{\&} \mathrm{Fe}$ \\
\hline$N_{\text {dim }}$ & 155 & 146 & 205 & 96 \\
$P_{\text {affil }}$ & 0.006 & 0.007 & 0.005 & 0.010 \\
\hline$N_{\text {coin }}(\mathrm{CDAW})$ & 59.5 & 55.3 & 72.5 & 42.2 \\
$P_{0}$ & 0.681 & 0.684 & 0.701 & 0.643 \\
$P_{\text {assoc }}$ & 0.319 & 0.316 & 0.299 & 0.357 \\
$N_{\text {assoc }}$ & 49.5 & 46.1 & 61.2 & 34.3 \\
$\sigma_{\text {assoc }}$ & 5.8 & 5.6 & 6.6 & 4.7 \\
$e_{\text {assoc }}$ & 3.8 & 3.8 & 3.9 & 3.4 \\
$\delta_{\text {assoc }}$ & 6.9 & 6.8 & 7.6 & 5.8 \\
$N_{\text {as-obs }}$ & 71 & 60 & 85 & 46 \\
$N_{\text {assoc }}(\%)$ & 32 & 32 & 30 & 36 \\
$\delta_{\text {assoc }}(\%)$ & 4 & 5 & 4 & 6 \\
$N_{\text {as-obs }}(\%)$ & 46 & 41 & 41 & 48 \\
\hline$N_{\text {coin }}(\mathrm{CACTus})$ & 85.2 & 79.1 & 103.8 & 60.4 \\
$P_{0}$ & 0.576 & 0.581 & 0.602 & 0.531 \\
$P_{\text {assoc }}$ & 0.424 & 0.419 & 0.398 & 0.469 \\
$N_{\text {assoc }}$ & 65.7 & 61.2 & 81.6 & 45.0 \\
$\sigma_{\text {assoc }}$ & 6.2 & 6.0 & 7.0 & 4.9 \\
$e_{\text {assoc }}$ & 3.9 & 3.9 & 4.0 & 3.4 \\
$\delta_{\text {assoc }}$ & 7.3 & 7.1 & 8.1 & 5.9 \\
$N_{\text {as-obs }}$ & 79 & 72 & 98 & 53 \\
$N_{\text {assoc }}(\%)$ & 42 & 42 & 40 & 47 \\
$\delta_{\text {assoc }}(\%)$ & 5 & 5 & 4 & 6 \\
$N_{\text {as-obs }}(\%)$ & 51 & 49 & 48 & 55 \\
\hline & & & &
\end{tabular}

\section{Conclusions and discussion}

It was the purpose of this report to demonstrate, statistically, - indeed, to confirm - the CME-dimming association. Such confirmation, then, highlights the possibility for utilising the detection of such dimming to predict the onset of a CME.

Thus, we have explored statistically the association between CME onsets and dimming events in two coronal emission lines, representing temperatures of 1 million $\mathrm{K}$ and 2 million $\mathrm{K}$. Indeed, we believe this is the first thorough statistical analysis of the CME association with coronal dimming and feel that this is long overdue given the numbers of papers discussing the dimming phenomenon in the context of CME onsets.

Our key results are the following:

- Up to $48 \%$ of dimming regions are associated with a CME in the CDAW catalogue and up to $55 \%$ are associated with a CME in the CACTus catalogue. When we compare this to a probability model of the number of random coincidences assuming that dimming regions and CMEs are not linked, we find that in most cases, the observed number of dimming region-CME associations are significantly greater (at the two standard deviation level) than the predicted number from the probability model. This is below the $2.25 \%$ confidence level (i.e., there is less that $2.25 \%$ chance of getting this result). Three cases (from CACTus) failed to reach this level of significance, but were significant to the 1.3 standard deviation level (or below the $9 \%$ confidence level). This strongly suggests that dimming region - CME associations are not random coincidences and that there is a link between the two phenomena.

- Up to $84 \%$ of CMEs associated with our data from the CDAW catalogue and up to $73 \%$ of CMEs from the CACTus catalogue can be tracked back to dimming regions in either 
the $\mathrm{Mg}$ or Fe lines. This compares to up to $58 \%$ in the random case and is significant to over 2 standard deviations. This strongly indicates that CMEs originate from regions that dim.

It is clear that the analysis in more than one emission line reveals more dimming events; monitoring in one emission line, or temperature, may not be sufficient for an effective prediction capability. The Mg IX and Fe XVI lines used here are clearly good dimming indicators but the analysis suggests that simultaneous monitoring across a broad range of lines in the range from 1 million $\mathrm{K}$ to a few million $\mathrm{K}$ would be the best approach.

We must remember that this work has been done using only limb events. There are bound to be occulted dimming events when dealing with limb regions and so some of the figures in this paper should be considered as lower limits. CME prediction on the disc, including Earth-directed CME events, is also feasible using such techniques even in the absence of associated coronagraph instrumentation.

A coronal dimming is a signature of the disappearance of material from the solar corona. There are many ways that this disappearance could occur and the following are 3 examples;

- The material could erupt and end up as a CME.

- The material could move out of the field-of-view.

- The material could cool or heat up passing out of the passband observed.

In this study, we have not distinguished between these different mechanisms, and so it is understandable why some of the dimming regions identified have CMEs associated and others don't.

Now that we have demonstrated the link between dimming regions and CMEs, further questions should be posed:

- Do different properties of the dimming region (for example, area) have a stronger relationship with CMEs. For example, are large dimming regions more likely to have a CME? Can these be used as extra criteria to identify dimming regions related to CMEs?

- Can we develop an algorithm to monitor for coronal dimming in EUV data and thus predict subsequent CME activity? Can we use the extra criteria in question 2 to reduce type I errors (where a dimming region is predicted to have a
CME, but does not) and/or type II errors (where a dimming region is not predicted to have a CME, but does)?

These questions will be addressed in future papers.

Acknowledgements. SOHO is a mission of international cooperation between ESA and NASA.

CDS was built and is operated by a consortium led by the Rutherford Appleton Laboratory and including the Mullard Space Science Laboratory, the NASA Goddard Space Flight Center, Oslo University and the Max-Planck-Institute for Extraterrestrial Physics, Garching.

The CDAW CME list is generated and maintained at the CDAW Data Center by NASA and The Catholic University of America in cooperation with the Naval Research Laboratory.

The CACTus CME catalogue uses an automated algorithm to identify CMEs in SOHO/LASCO data and is maintained by the SIDC - Royal Observatory of Belgium.

DSB would like to thank the UK's Science and Technology Facilities Council, formerly the Particle Physics and Astronomy Research Council, for financial support.

\section{References}

Bewsher, D., \& Harrison, R. A. 2006, ESA SP-617, 21.1

Biesecker, D. A., Myers, D. C., Thompson, B. J., et al. 2002, ApJ, 569, 1009

Brueckner, G. E., Howard, R. A., Koomen, M. J., et al. 1995, Sol. Phys., 162, 357

Gopalswamy, N., \& Hanaoka, Y. 1998, ApJ, 498, 179

Harrison, R. A. 1997, Proc. 31st ESLAB Symp., Correlated Phenomena at the Sun, in the Heliosphere and in Geospace, ESA SP-415, 121

Harrison, R. A. 2003, Adv. Space Res. 32, No. 12, 2425

Harrison, R. A. 2006, AGU Monograph Solar Coronal Mass Ejections and Energetic Particles, 165, 73

Harrison, R. A., \& Lyons, M. 2000, A\&A, 358, 1097

Harrison, R. A., \& Bewsher, D. 2007, A\&A, 461, 1155

Harrison, R. A., Sawyer, E. C., Carter, M. K., et al. 1995, Sol. Phys., 162, 233

Harrison, R. A., Bryans, P., Simnett, G. M., \& Lyons, M. 2003, A\&A, 400, 1071

Howard, T. A., \& Harrison, R. A. 2004, Sol. Phys., 219, 315

Hudson, H. S., \& Webb, D. F. 1997, AGU Monograph Coronal Mass Ejections, 99, 27

Robbrecht, E., \& Berghmans, D. 2004, A\&A, 425, 1097

Rust, D. M. 1983, Space Sci. Rev., 34, 21

Rust, D. M., \& Hildner, E. 1976, Sol. Phys., 48, 381

Sterling, A. C., \& Hudson, H. S. 1997, ApJ, 491, L55

Thompson, W. 1998, CDS Software note, No. 49

Watanabe, T., Kozuka, Y., Ohyama, M., et al. 1992, PASJ, 44, L199

Zarro, D. M., Sterling, A. C., Thompson, B. J., et al. 1999, ApJ, 520, 139 
D. Bewsher et al.: The relationship between EUV dimming and coronal mass ejections. I., Online Material $p 1$

\section{Online Material}


D. Bewsher et al.: The relationship between EUV dimming and coronal mass ejections. I., Online Material p 2

\section{Appendix A: CDS observations}

Table A.1 provides a complete list of the CDS observations used in this statistical study of coronal dimmings. For each row we identify a CDS CME onset (JOP 67) campaign period, defining the CDS study number, the date and times of the sequence, the number of rasters and the pointing locations of those rasters in arcseconds (north and west being positive). 
Table A.1. List of the CDS observations used in the study.

\begin{tabular}{|c|c|c|c|c|}
\hline CDS study number & Date & $\overline{\text { Times }}$ & No. of rasters & Positions \\
\hline 10322 & 1998-01-29 & $07: 23: 36-13: 46: 32$ & 24 & $(939,-56),(931,-298),(935,-541)$ \\
\hline 10330 & 1998-01-30 & $08: 14: 28-13: 47: 21$ & 21 & $(932,-60),(931,-297),(935,-541)$ \\
\hline 10386 & 1998-02-10 & $06: 53: 25-16: 36: 15$ & 36 & $(1000,-83),(998,-302),(999,-522)$ \\
\hline 10400 & 1998-02-11 & $06: 51: 19-16: 34: 02$ & 36 & $(1000,-84),(998,-302),(999,-523)$ \\
\hline 10407 & 1998-02-12 & $06: 52: 10-15: 42: 52$ & 33 & $(-904,601),(-972,396),(-973,179)$ \\
\hline 10415 & 1998-02-13 & $06: 51: 09-15: 39: 53$ & 33 & $(-954,-380),(-878,-596),(-699,-711)$ \\
\hline 15359 & $1998-12-15$ & $06: 18: 42-13: 31: 35$ & 27 & $(-1000,241),(-1002,-4),(-1001,-247)$ \\
\hline 15366 & 1998-12-16 & $06: 50: 47-14: 03: 36$ & 27 & $(-1000,242),(-1000,-2),(-1002,-246)$ \\
\hline 15369 & 1998-12-17 & 07:00:29-15:03:17 & 30 & $(-999,241),(-1002,-3),(-1001,-247)$ \\
\hline 15377 & $1998-12-18$ & $07: 00: 28-14: 13: 21$ & 27 & $(-999,243),(-1002,-3),(-999,-244)$ \\
\hline 15379 & 1998-12-19 & $07: 00: 30-12: 33: 23$ & 21 & $(-999,241),(-1002,-3),(-1000,-244)$ \\
\hline $15555^{2}$ & 1999-02-14 & $00: 10: 36-11: 33: 23$ & 42 & $(-762,712),(-879,514),(-994,317)$ \\
\hline 15607 & 1999-03-01 & $18: 12: 58-22: 55: 57$ & 18 & $(-761,-638),(-880,-427),(-994,-216)$ \\
\hline 15610 & 1999-03-02 & $07: 36: 38-12: 19: 35$ & 18 & $(-761,-638),(-880,-428),(-995,-217)$ \\
\hline 15688 & 1999-03-20 & $16: 16: 55-23: 25: 12$ & 27 & $(-666,783),(-795,637),(-919,458)$ \\
\hline 15796 & 1999-04-06 & $14: 44: 55-23: 33: 32$ & 33 & $(-804,663),(-911,485),(-940,286)$ \\
\hline 15801 & 1999-04-07 & 18:04:07-23:34:30 & 21 & $(-802,661),(-911,486),(-940,286)$ \\
\hline 15839 & 1999-04-16 & $12: 13: 26-22: 41: 51$ & 39 & $(803,633),(910,427),(997,220)$ \\
\hline 15866 & 1999-04-20 & $16: 43: 12-23: 03: 29$ & 24 & $(978,-317),(874,-536),(743,-677)$ \\
\hline 15874 & 1999-04-21 & $18: 22: 11-23: 52: 38$ & 21 & $(982,-265),(890,-472),(803,-649)$ \\
\hline 15969 & 1999-05-04 & $18: 45: 47-23: 25: 52$ & 18 & $(847,556),(922,395),(975,215)$ \\
\hline 15991 & 1999-05-05 & 19:04:45-23:44:01 & 18 & $(864,518),(948,394),(975,215)$ \\
\hline 15997 & 1999-05-06 & 19:07:20-23:47:52 & 18 & $(-950,376),(-957,196),(-974,16)$ \\
\hline 16020 & 1999-05-07 & 19:05:57-23:46:44 & 18 & $(-981,-64),(-978,-260),(-896,-457)$ \\
\hline 16023 & 1999-05-08 & $21: 30: 08-01: 20: 41^{1}$ & 15 & $(-945,460),(-957,288),(-977,111)$ \\
\hline 16035 & 1999-05-10 & 08:05:29-14:24:22 & 24 & $(840,424),(888,278),(908,97)$ \\
\hline 16641 & 1999-06-26 & $07: 32: 36-13: 05: 31$ & 21 & $(-1001,239),(-1000,-4),(-999,-242)$ \\
\hline 16725 & 1999-07-04 & $06: 56: 36-15: 43: 14$ & 33 & $(902,552),(948,413),(999,239)$ \\
\hline 16767 & 1999-07-10 & 07:06:05-19:10:41 & 45 & $(909,-532),(968,-386),(1006,-215)$ \\
\hline 16855 & 1999-07-18 & 09:58:12-14:41:04 & 18 & $(-943,402),(-847,622),(-675,848)$ \\
\hline 17048 & 1999-08-01 & 10:03:17-19:42:55 & 36 & $(866,576),(954,370),(1020,162)$ \\
\hline 17148 & 1999-08-08 & 09:58:49-17:58:12 & 30 & $(860,-600),(943,-396),(1018,-196)$ \\
\hline 18193 & 1999-12-18 & $07: 02: 38-12: 35: 32$ & 21 & $(-999,242),(-999,-3),(-1000,-243)$ \\
\hline 18447 & 2000-01-22 & $15: 03: 30-23: 52: 08$ & 33 & $(1015,-46),(979,-243),(928,-431)$ \\
\hline 18456 & 2000-01-24 & $06: 44: 23-15: 32: 57$ & 33 & $(1010,-50),(980,-242),(929,-430)$ \\
\hline $18539^{2}$ & $2000-02-08$ & $15: 32: 13-23: 31: 19$ & 30 & $(-1032,-103),(-976,-302),(-911,-476)$ \\
\hline 18560 & 2000-02-09 & $16: 14: 52-23: 24: 25$ & 27 & $(-1034,-90),(-979,-291),(-916,-464)$ \\
\hline 18597 & 2000-02-15 & $06: 57: 51-16: 37: 35$ & 36 & $(1034,-200),(968,-417),(866,-590)$ \\
\hline 18601 & 2000-02-16 & $06: 39: 33-16: 19: 17$ & 36 & $(1034,-200),(968,-417),(866,-590)$ \\
\hline 18748 & 2000-03-03 & $06: 39: 26-20: 27: 40$ & 51 & $(1058,-118),(999,-328),(901,-530)$ \\
\hline 19417 & 2000-04-24 & 09:41:11-17:38:07 & 30 & $(955,385),(892,577),(769,698)$ \\
\hline 19437 & 2000-04-26 & $16: 41: 49-00: 39: 30^{1}$ & 30 & $(625,768),(745,606),(856,448)$ \\
\hline 19460 & 2000-04-30 & 18:03:39-23:34:35 & 21 & $(971,-208),(888,-407),(974,-13)$ \\
\hline 19714 & $2000-05-27$ & 07:08:45-17:36:42 & 39 & $(896,563),(949,381),(1021,175)$ \\
\hline 19809 & 2000-06-11 & $12: 32: 49-23: 00: 52$ & 39 & $(-905,459),(-970,261),(-1011,64)$ \\
\hline 19874 & $2000-06-21$ & $08: 10: 32-22: 53: 12$ & 54 & $(949,528),(951,288),(950,49)$ \\
\hline 19972 & 2000-07-02 & $14: 38: 55-22: 40: 45$ & 30 & $(1006,319),(1009,102),(1008,-114)$ \\
\hline 20033 & 2000-07-09 & $13: 51: 12-23: 28: 23$ & 36 & $(-933,436),(-802,617),(-693,795)$ \\
\hline 20053 & $2000-07-12$ & 06:48:42-18:06:22 & 42 & $(981,-176),(942,-371),(867,-569)$ \\
\hline 20071 & $2000-07-14$ & $07: 37: 07-15: 36: 21$ & 30 & $(907,501),(958,335),(1011,129)$ \\
\hline 20209 & $2000-08-05$ & $06: 51: 39-17: 22: 16$ & 39 & $(-1052,44),(-1008,257),(-893,476)$ \\
\hline 20292 & 2000-08-19 & $06: 51: 19-17: 17: 21$ & 39 & $(849,-529),(724,-712),(605,-860)$ \\
\hline 20371 & $2000-08-26$ & $06: 31: 42-17: 04: 30$ & 39 & $(1000,240),(1000,-4),(997,-244)$ \\
\hline 20507 & $2000-09-10$ & $12: 51: 15-20: 55: 23$ & 30 & $(-1052,78),(-1026,300),(-954,522)$ \\
\hline 21079 & $2000-10-22$ & $12: 25: 31-23: 03: 22$ & 39 & $(-1000,239),(-1000,-6),(-997,-245)$ \\
\hline 21581 & $2000-12-17$ & 12:19:07-22:52:06 & 39 & $(-956,536),(-1016,318),(-1082,112)$ \\
\hline 21658 & 2001-01-05 & $16: 34: 23-22: 08: 06$ & 21 & $(-910,552),(-1010,328),(-1061,110)$ \\
\hline
\end{tabular}


Table A.1. continued.

\begin{tabular}{|c|c|c|c|c|}
\hline CDS study number & Date & $\overline{\text { Times }}$ & No. of rasters & Positions \\
\hline 21666 & 2001-01-07 & $12: 42: 26-23: 16: 48$ & 39 & $(-910,553),(-1010,329),(-1062,109)$ \\
\hline 21737 & 2001-01-20 & $06: 30: 21-05: 30: 48^{1}$ & 84 & $(944,436),(1016,256),(1050,75)$ \\
\hline 21822 & 2001-02-04 & $12: 13: 23-22: 53: 09$ & 39 & $(-1032,212),(-1034,461),(-1032,-36)$ \\
\hline 21865 & 2001-02-11 & $11: 49: 16-22: 20: 37$ & 39 & $(712,801),(829,627),(941,426)$ \\
\hline 21870 & 2001-02-12 & $13: 12: 26-23: 43: 57$ & 39 & $(711,801),(828,626),(942,427)$ \\
\hline 22125 & 2001-04-08 & 00:02:15-22:57:02 & 84 & $(843,538),(937,379),(997,204)$ \\
\hline 22182 & 2001-04-16 & $23: 45: 11-23: 26: 09^{1}$ & 87 & $(1013,-251),(947,-411),(873,-556)$ \\
\hline 22205 & 2001-04-22 & $12: 24: 42-23: 40: 32$ & 42 & $(-1032,299),(-937,452),(-828,594)$ \\
\hline 22246 & 2001-04-29 & $12: 34: 59-23: 10: 03$ & 39 & $(1035,185),(973,419),(865,647)$ \\
\hline 22299 & 2001-05-06 & $12: 43: 41-23: 16: 29$ & 39 & $(987,389),(1033,201),(1032,4)$ \\
\hline 22349 & 2001-05-11 & $12: 51: 03-23: 19: 02$ & 39 & $(846,664),(905,519),(982,349)$ \\
\hline 22529 & 2001-05-29 & $16: 02: 19-23: 15: 20$ & 27 & $(915,459),(992,269),(1053,49)$ \\
\hline 22569 & 2001-06-03 & $12: 43: 41-23: 16: 28$ & 39 & $(987,389),(1032,200),(1033,3)$ \\
\hline 22606 & 2001-06-07 & $14: 03: 24-23: 43: 05$ & 36 & $(-861,470),(-969,328),(-1021,144)$ \\
\hline 22613 & 2001-06-08 & $14: 08: 25-22: 55: 43$ & 33 & $(776,587),(879,455),(974,308)$ \\
\hline 22615 & 2001-06-09 & 04:03:33-12:50:49 & 33 & $(783,589),(892,455),(974,308)$ \\
\hline 22625 & 2001-06-10 & $14: 02: 24-22: 52: 23$ & 33 & $(1009,-4),(1007,-158),(985,-343)$ \\
\hline 22682 & 2001-06-17 & $07: 01: 12-23: 26: 20$ & 60 & $(901,512),(963,297),(1020,78)$ \\
\hline 22722 & 2001-06-24 & $12: 45: 30-23: 19: 56$ & 39 & $(890,500),(980,279),(1010,67)$ \\
\hline 22855 & 2001-07-15 & $06: 27: 24-13: 40: 42$ & 27 & $(1002,6),(1000,-238),(1001,248)$ \\
\hline 22906 & 2001-07-22 & $13: 23: 02-23: 06: 20$ & 36 & $(1002,3),(999,-240),(1003,245)$ \\
\hline 23290 & 2001-09-09 & $06: 47: 49-23: 10: 20$ & 60 & $(1000,241),(1001,15),(998,-202)$ \\
\hline 23336 & 2001-09-16 & $12: 22: 48-22: 49: 26$ & 39 & $(786,-586),(917,-424),(1011,-232)$ \\
\hline 23389 & $2001-09-23$ & $12: 13: 48-22: 43: 17$ & 39 & $(-1003,287),(-1046,89),(-1043,-125)$ \\
\hline 23405 & 2001-09-29 & 07:04:30-15:57:18 & 33 & $(1032,249),(1030,28),(1029,-192)$ \\
\hline 23479 & 2001-10-07 & $13: 17: 43-22: 57: 02$ & 36 & $(964,410),(1000,208),(1028,2)$ \\
\hline 23515 & $2001-10-11$ & $23: 38: 17-23: 19: 01^{1}$ & 87 & $(-997,417),(-1036,247),(-1068,57)$ \\
\hline $23668^{2}$ & 2001-11-04 & $12: 24: 03-22: 50: 32$ & 39 & $(1025,21),(1018,189),(969,378)$ \\
\hline 23709 & 2001-11-11 & $12: 24: 56-22: 58: 05$ & 39 & $(-1034,6),(-1030,243),(-1032,-232)$ \\
\hline 23746 & 2001-11-18 & $11: 56: 45-22: 29: 35$ & 39 & $(980,-488),(1060,-261),(1070,-36)$ \\
\hline 23857 & 2001-12-09 & $07: 10: 53-17: 43: 39$ & 39 & $(1042,220),(1071,-1),(1041,-223)$ \\
\hline 24181 & 2002-02-19 & $06: 58: 06-22: 25: 16$ & 57 & $(970,467),(1027,260),(1064,59)$ \\
\hline 24214 & $2002-02-24$ & $11: 36: 56-22: 53: 01$ & 42 & $(964,524),(1015,347),(1053,170)$ \\
\hline 24342 & 2002-03-10 & $11: 46: 18-23: 05: 32$ & 42 & $(1020,176),(1044,-19),(1003,-249)$ \\
\hline 24508 & $2002-03-31$ & $12: 23: 36-22: 51: 26$ & 39 & $(991,332),(1030,138),(1055,-61)$ \\
\hline 24905 & 2002-05-19 & 11:51:08-22:23:54 & 39 & $(-1017,411),(-1019,176),(-1018,-54)$ \\
\hline $25009^{2}$ & 2002-06-01 & 07:30:04-18:02:50 & 39 & $(1000,360),(1000,139),(999,-84)$ \\
\hline 25226 & $2002-06-23$ & $12: 25: 23-22: 58: 10$ & 39 & $(1000,250),(931,469),(796,687)$ \\
\hline 25267 & $2002-06-30$ & $12: 37: 49-23: 05: 23$ & 39 & $(1024,4),(1023,-183),(959,-359)$ \\
\hline 25324 & $2002-07-10$ & $09: 50: 54-22: 53: 33$ & 48 & $(-996,364),(-1011,146),(-1033,-80)$ \\
\hline 25377 & $2002-07-22$ & 00:01:35-22:53:05 & 84 & $(919,441),(966,246),(1020,52)$ \\
\hline 25417 & $2002-07-27$ & 15:19:07-20:49:28 & 21 & $(1008,0),(1003,-198),(961,-361)$ \\
\hline 25542 & $2002-08-10$ & 05:07:58-13:07:00 & 30 & $(-920,443),(-967,246),(-1021,50)$ \\
\hline 25832 & 2002-09-15 & $12: 32: 25-23: 05: 15$ & 39 & $(-1000,241),(-1000,-2),(-999,-242)$ \\
\hline 25880 & $2002-09-22$ & $12: 26: 32-22: 52: 56$ & 39 & $(915,438),(972,263),(1004,84)$ \\
\hline 25902 & 2002-09-26 & $06: 59: 48-22: 32: 28$ & 57 & $(1053,-60),(1037,-292),(957,-523)$ \\
\hline 25906 & 2002-09-27 & $10: 32: 45-22: 45: 25$ & 45 & $(1054,-59),(1037,-292),(957,-523)$ \\
\hline 25909 & $2002-09-28$ & $08: 10: 15-22: 52: 55$ & 54 & $(1059,-53),(1037,-292),(957,-523)$ \\
\hline 25915 & 2002-09-30 & 07:00:52-19:58:20 & 48 & $(985,-341),(1012,-139),(1031,64)$ \\
\hline 25997 & $2002-10-15$ & 06:03:01-23:04:56 & 63 & $(915,438),(973,265),(1003,84)$ \\
\hline 26033 & $2002-10-19$ & 07:02:48-15:50:19 & 33 & $(915,438),(973,264),(1004,83)$ \\
\hline 26285 & $2002-11-20$ & $13: 03: 12-01: 12: 52^{1}$ & 45 & $(1051,-157),(998,-379),(907,-583)$ \\
\hline 26304 & $2002-11-23$ & $08: 17: 55-20: 27: 53$ & 45 & $(1054,-154),(999,-379),(907,-583)$ \\
\hline 26341 & $2002-11-28$ & $09: 56: 17-22: 52: 43$ & 48 & $(1047,-152),(955,-346),(865,-522)$ \\
\hline 26359 & $2002-12-01$ & $12: 39: 36-16: 30: 41$ & 15 & $(989,281),(914,475),(809,643)$ \\
\hline 26360 & $2002-12-01$ & 19:02:37-23:43:12 & 18 & $(987,281),(913,475),(809,643)$ \\
\hline 26464 & $2002-12-15$ & $12: 53: 27-23: 21: 41$ & 39 & $(1053,139),(990,338),(916,530)$ \\
\hline 26596 & 2003-01-05 & $11: 08: 25-22: 23: 56$ & 42 & $(913,486),(985,315),(1028,153)$ \\
\hline 26649 & 2003-01-12 & $11: 04: 22-22: 25: 02$ & 42 & $(-840,702),(-960,486),(-1032,277)$ \\
\hline
\end{tabular}


D. Bewsher et al.: The relationship between EUV dimming and coronal mass ejections. I., Online Material p 5

Table A.1. continued.

\begin{tabular}{|c|c|c|c|c|}
\hline CDS study number & Date & $\overline{\text { Times }}$ & No. of rasters & Positions \\
\hline 26741 & $2003-01-21$ & $11: 15: 17-23: 25: 12$ & 45 & $(850,-589),(947,-379),(1008,-169)$ \\
\hline 26775 & 2003-01-25 & $07: 25: 19-17: 53: 26$ & 39 & $(1058,-20),(1041,181),(1010,365)$ \\
\hline 26824 & 2003-02-02 & $12: 32: 17-23: 52: 21$ & 42 & $(1062,98),(1071,-113),(999,-322)$ \\
\hline 26907 & 2003-02-17 & $06: 50: 48-22: 18: 27$ & 57 & $(941,490),(1020,302),(1064,83)$ \\
\hline 26996 & 2003-02-28 & $17: 14: 38-23: 32: 33$ & 24 & $(1002,315),(961,448),(870,616)$ \\
\hline 27001 & 2003-03-01 & $11: 45: 48-18: 05: 41$ & 24 & $(1046,189),(997,370),(888,553)$ \\
\hline 27069 & 2003-03-09 & $12: 38: 23-23: 08: 54$ & 39 & $(-941,490),(-1031,267),(-1062,58)$ \\
\hline 27164 & 2003-03-23 & $12: 11: 06-21: 53: 55$ & 36 & $(-941,491),(-1033,267),(-1062,45)$ \\
\hline 27194 & 2003-03-30 & $12: 58: 38-23: 31: 59$ & 39 & $(-975,319),(-1012,70),(-1002,-174)$ \\
\hline 27249 & 2003-04-06 & $12: 37: 34-23: 10: 45$ & 39 & $(973,321),(1010,72),(1001,-175)$ \\
\hline 27286 & 2003-04-13 & $12: 30: 31-22: 59: 26$ & 39 & $(-1001,277),(-1051,68),(-1001,-142)$ \\
\hline 27439 & 2003-04-27 & $15: 28: 14-23: 27: 32$ & 30 & $(881,561),(945,363),(1018,163)$ \\
\hline 27622 & 2003-05-11 & $12: 38: 39-23: 06: 27$ & 39 & $(-938,517),(-1023,310),(-1065,104)$ \\
\hline 27849 & 2003-06-08 & $13: 10: 44-22: 53: 33$ & 36 & $(-853,-652),(-949,-436),(-1029,-212)$ \\
\hline 27974 & 2003-07-13 & 13:19:30-23:02:15 & 36 & $(989,383),(1032,144),(1031,-99)$ \\
\hline 28101 & 2003-07-27 & $12: 23: 35-22: 51: 28$ & 39 & $(-968,280),(-1003,99),(-1005,-109)$ \\
\hline 28167 & 2003-08-03 & $12: 44: 07-23: 12: 10$ & 39 & $(1019,2),(1007,185),(946,386)$ \\
\hline 28410 & 2003-08-31 & $12: 36: 35-23: 05: 20$ & 39 & $(935,-417),(992,-215),(991,-8)$ \\
\hline 28556 & 2003-09-20 & 07:04:57-18:27:41 & 42 & $(-1050,70),(-1006,-157),(-971,-384)$ \\
\hline 28640 & 2003-10-05 & $11: 40: 55-22: 58: 58$ & 42 & $(1006,-172),(939,-382),(824,-542)$ \\
\hline 28691 & 2003-10-12 & $12: 07: 42-22: 34: 11$ & 39 & $(1044,-72),(1010,-244),(942,-424)$ \\
\hline 28837 & 2003-11-02 & 11:59:33-22:30:10 & 39 & $(1084,-78),(990,-290),(928,-507)$ \\
\hline 29182 & 2003-12-11 & 11:54:38-23:11:57 & 42 & $(944,-375),(991,-182),(1027,2)$ \\
\hline 29217 & 2003-12-16 & $07: 45: 07-22: 27: 47$ & 54 & $(-1062,218),(-1081,-3),(-1063,-225)$ \\
\hline 29309 & 2004-01-10 & 07:21:29-17:01:11 & 36 & $(1047,-242),(1041,-44),(1038,171)$ \\
\hline 29497 & 2004-02-07 & $06: 29: 06-22: 01: 42$ & 57 & $(1050,221),(1080,1),(1050,-221)$ \\
\hline 29499 & 2004-02-08 & $06: 40: 06-18: 02: 46$ & 42 & $(1049,221),(1080,1),(1050,-221)$ \\
\hline 29502 & 2004-02-09 & $06: 38: 23-22: 10: 58$ & 57 & $(1051,222),(1081,1),(1050,-221)$ \\
\hline 29602 & 2004-02-22 & 13:00:55-18:31:08 & 21 & $(974,-387),(1021,-191),(1040,-23)$ \\
\hline 29605 & $2004-02-23$ & $06: 38: 18-22: 10: 54$ & 57 & $(-1050,233),(-1051,0),(-1050,-230)$ \\
\hline 29607 & 2004-02-24 & $06: 39: 26-22: 12: 08$ & 57 & $(-1051,233),(-1050,0),(-1051,-231)$ \\
\hline 29614 & 2004-02-25 & $06: 45: 38-22: 18: 18$ & 57 & $(-1059,224),(-1050,0),(-1050,-230)$ \\
\hline 29616 & 2004-02-26 & $06: 39: 47-22: 12: 28$ & 57 & $(-1051,233),(-1050,0),(-1051,-231)$ \\
\hline 29684 & 2004-03-07 & $12: 46: 40-19: 05: 58$ & 24 & $(-1017,-87),(-985,-255),(-936,-444)$ \\
\hline 29749 & 2004-03-14 & $12: 45: 31-16: 38: 28$ & 15 & $(1067,158),(1066,-70),(1071,-306)$ \\
\hline 29907 & 2004-04-11 & $12: 24: 51-18: 47: 33$ & 24 & $(1065,-120),(1024,-342),(933,-554)$ \\
\hline 30056 & 2004-05-09 & $12: 24: 20-22: 57: 09$ & 39 & $(-1043,138),(-1040,-101),(-1041,-333)$ \\
\hline 30193 & 2004-05-29 & $07: 22: 52-22: 55: 26$ & 57 & $(-1052,13),(-1041,-207),(-972,-428)$ \\
\hline 30195 & 2004-05-30 & $06: 29: 50-22: 52: 24$ & 60 & $(-1052,13),(-1041,-207),(-972,-428)$ \\
\hline 30197 & 2004-05-31 & $07: 01: 38-22: 34: 10$ & 57 & $(-1052,13),(-1041,-207),(-972,-428)$ \\
\hline $30419^{2}$ & 2004-07-03 & $06: 59: 18-21: 41: 59$ & 54 & $(-1035,286),(-1029,69),(-1031,-149)$ \\
\hline $30421^{2}$ & 2004-07-04 & $06: 29: 27-17: 52: 05$ & 42 & $(-1029,292),(-1029,69),(-1031,-149)$ \\
\hline 30891 & 2004-08-21 & 09:01:01-18:43:46 & 36 & $(-1013,301),(-1012,61),(-1013,-180)$ \\
\hline 31002 & 2004-09-04 & 14:22:03-23:14:51 & 33 & $(-1001,-240),(-999,3),(-998,242)$ \\
\hline 31799 & 2005-01-02 & $11: 46: 27-22: 19: 12$ & 39 & $(-929,350),(-927,100),(-929,-146)$ \\
\hline 31843 & 2005-01-09 & 11:45:51-23:08:39 & 42 & $(-1025,136),(-1018,-79),(-1017,-300)$ \\
\hline 31912 & 2005-01-22 & $06: 52: 51-22: 25: 33$ & 57 & $(1007,376),(1006,138),(1007,-103)$ \\
\hline 32058 & $2005-02-13$ & 12:41:22-19:04:10 & 24 & $(-1065,233),(-1087,9),(-1074,-209)$ \\
\hline 32278 & $2005-03-26$ & $06: 27: 39-17: 00: 26$ & 39 & $(1000,241),(999,-3),(997,-243)$ \\
\hline 32333 & 2005-04-03 & $12: 36: 21-18: 55: 16$ & 24 & $(934,364),(997,203),(1035,55)$ \\
\hline 32634 & 2005-05-15 & $14: 11: 24-22: 14: 12$ & 30 & $(1001,240),(1000,-2),(998,-242)$ \\
\hline 32859 & 2005-06-26 & $12: 26: 55-23: 43: 22$ & 42 & $(1022,-140),(960,-331),(918,-497)$ \\
\hline 33886 & $2005-11-20$ & $12: 41: 58-22: 22: 56$ & 36 & $(-890,-374),(-793,-595),(-691,-801)$ \\
\hline 33970 & 2005-11-27 & $12: 15: 57-23: 38: 38$ & 42 & $(1037,49),(984,-188),(930,-425)$ \\
\hline 33972 & $2005-11-28$ & $07: 42: 10-23: 14: 49$ & 57 & $(1037,49),(984,-187),(930,-425)$ \\
\hline 34060 & $2005-12-18$ & $12: 32: 41-23: 00: 41$ & 39 & $(1055,3),(1018,195),(970,398)$ \\
\hline 34107 & $2005-12-24$ & 07:00:50-17:30:46 & 39 & $(-1019,177),(-1034,-34),(-1007,-249)$ \\
\hline 34111 & $2005-12-25$ & $12: 24: 30-22: 52: 54$ & 39 & $(-1004,134),(-1018,-70),(-1004,-270)$ \\
\hline
\end{tabular}

$1+1$ day.

${ }^{2}$ No LASCO data. 